\title{
Analyzing Wavelet and Bidimensional Empirical Mode Decomposition of MRI Segmentation using Fuzzy C-Means Clustering
}

\author{
Gulam Sarwar Chuwdhury ${ }^{1 *}$, Md. Khaliluzzaman ${ }^{2}$ \\ and Md. Rashed-Al-Mahfuz ${ }^{3}$ \\ ${ }^{1,2}$ Department of Computer Science and Engineering, International Islamic \\ University Chittagong, Chittagong 4203, Bangladesh. \\ ${ }^{3}$ Department of Computer Science and Engineering, University of Rajshahi, \\ Rajshahi 6205, Bangladesh.
}

*Corresponding Author: khalil_021@yahoo.co.in²

\begin{abstract}
Image segmentation is a vital step in medical image processing. Magnetic resonance imaging (MRI) is used for brain tissues extraction in white and gray matter. These tissues extraction help in image segmentation applications such as radiotherapy planning, clinical diagnosis, treatment planning. This paper presents utilization of fuzzy C-means (FCM) clustering by using wavelet and bidimensional empirical mode decomposition (BEMD) to improve the quality of noisy MR images. The signal to noise ratio (SNR) value is calculated from FCM clustering data to examine the best segmentation technique. The experiment with synthetic Brain Web images has demonstrated the efficiency and robustness of the appropriate approach in segmenting medical MRI.
\end{abstract}

Keywords - Image segmentation; fuzzy C-means; magnetic resonance imaging; wavelet; BEMD; SNR

INTRODUCTION

The joint space-spatial frequency representations have received spatial attention in the field of vision, image processing, and pattern recognition. Image segmentation from Magnetic Resonance (MR) image is such a challenging and significant step in medical image analysis. This step is essential in many medical image applications. Magnetic Resonance Imaging (MRI) is a noninvasive method for imaging internal tissues and organs. As MR images have difficult nature, also have no linear features. MR images performance is affected by many issues as partial volumes effects (PVE) which means a pixel contains more than one tissue, this leads to misclassification as a result of blurred boundary between artifact intensities of the same tissue are not constant over the image spatial domain and geometric deformation [5].

To satisfy the increasing requirement of image segmentation from MR images a variety of segmentation methods have been developed over past several years. The problems of image segmentation i.e. identification of tissues and organs of MR images have been described extensively in the literature and many algorithms have been developed in an attempt to solve the problems. One of the goals of segmentation of MR images is to determine the volumes of organs, tissues, and lesions present in a given patient. These volumes and the changes in these volumes over time, may aid in the diagnosis, prognosis and treatment planning of patients under investigation. Segmentation process also helps to find region of interest in a particular image. The key goal is to make image more simple and meaningful. 
There are different classifications of medical image segmentation techniques, however, no standard classification technique. The most commonly used segmentation techniques can be classified into many approaches, such as region based segmentation techniques that look for regions satisfying a given homogeneity criterion [6], basis of structural information of abnormal and normal tissues [2], edge-based segmentation techniques that look for edges between regions with different characteristics [7], artificial neural networks base method [8], data fusion based methods [1][9], Markov random field based methods [10] and hybrid based Methods [4].

Fuzzy C-means (FCM) is being employed for quite some time. A multi-resolution approach using discrete wavelet transform is employed on real time images for image segmentation in [3]. Features are extracted, selected and then finally segmented by Kmeans clustering algorithm. A method given in [11] proposes an MRI segmentation using neural network based on FCM clustering algorithm. The authors have performed experiment on one channel MR data, however, whereas MR images are multi-spectral and provides additional information; due to noise and in homogeneity this algorithm fails to work. The FCM based techniques in [12] employ Gaussian smoothing to produce a more homogeneous and low-noise subject to work with. This approach is, however, limited by the equal feature weights of the standard FCM. In [13], a modified FCM algorithm is given for MRI brain image segmentation using both local and non-local spatial constraints. This technique takes into account local and non-local spatial information using a variation index instead of typical distance metric. This approach has some limitation for its applicability to large 3D data and is computationally expensive. In [14], authors employ the FCM segmentation of MRI in a technique. By using the Gullied filter for pre-processing to remove in homogeneity in the images.

A method is introduced for MRI segmentation with Multi-modality image fusion in [1]. The main aim of this work is to improve cerebral MRI real images segmentation by fusion of modalities (T1, T2 and DP) using estimation the maximization Approach (EM). However, this method is not performed well with noisy MRI image. Another method is proposed for image segmentation using wavelet based multi-resolution Expectation Maximum (EM) algorithm in [15]. The drawback in EM is that it is based on identical and independent distribution of pixel intensities which may not be the case with noisy medical images. In [16], represent a method of Fuzzy C-Means clustering by using wavelet decomposition technique for features extraction. This method performs better for smooth MR images but not satisfactory for the noisy MR images. This method is nonadaptive to characterize textures by filter responses directly.

In [17], gives an overview of the state-of-the-art methods of EMD to decompose an image into a number of IMFs and a residue image with a minimum number of extrema points. In [18], authors developed an algorithm based on BEMD to extract features at multiple scales or spatial frequencies. This method derived data from image that are fully unsupervised and permits to analyze non-linear and non-stationary data as texture images. The BEMD technique is a fully data driven method, it does not use any pre-determine filter or wavelet functions. 
For MRI segmentation clustering is one of the most usable or utilizable technique, where it classifies pixels into classes, without knowing previous information or training. It classifies pixels with highest probability into the same class. It may find unclassified pixels which do not belong to any class probability. Clustering techniques training is done by using pixel features with properties of each class [19].

This paper has compared two image decomposition techniques i.e. wavelet and BEMD to determine the best technique which is used before the image clustering technique FCM. For that purpose, first apply FCM on decomposition image then select the best segmented technique from the two segmented approach through the value of SNR.

The paper is organized as follows. In Section II proposed method is described. In the next section experimental result is explained. The paper is concluded in Section IV.

\section{Proposed METHOD}

The proposed method is an efficient approach to segment the noisy MRI brain images. Two major stages are involved in proposed methodology i.e. feature extraction and clustering. Feature extraction process is performed by using 2D wavelet decomposition and BEMD. The wavelet decomposition outputs are low pass that is approximation component and high pass that is detailed components at horizontal, vertical, and diagonal. To obtain the wavelet features, here Dubechies-1(DAUB1) wavelet is applied to the images. Feature extraction from wavelet decomposition and BEMD are given to Fuzzy CMeans (FCM), FCM applied on the feature vector obtained from previous step for clustering. The output image will be segmented into two classes i.e. white matter and gray matter MRI images. Then calculate the SNR for segmented outputs. Finally, provide the output which gives the best SNR for MR images. The workflow of the proposed method is as shown in Fig. 1.

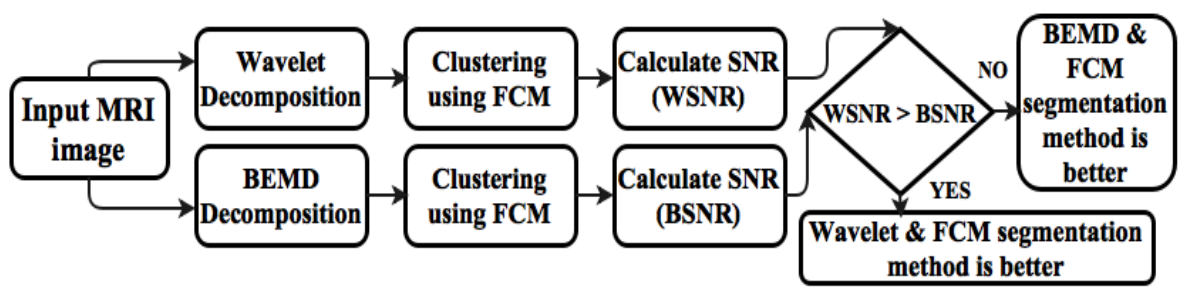

Fig. 1. Workflow of the proposed method.

\section{A. Wavelet Decomposition}

Although the Fourier transform has been mainstay of transform-based image processing, a more recent transformation, called the wavelet transform, is now making it even easier to compress, transmit and analyze MR images. Characteristics of Wavelet Transforms (WT) families share properties of their basis functions, primarily the finite support for the frequency and original domains, as well as the scalability. Wavelet families include Haar, Daubechies, Symlets, Coiflets, Biorthogonal, and Reverse biorthogonal, whereas in this paper Daubechies family is rather used. This family is one of the most used wavelet families in image and signal processing applications such as compression, de-noising, classification, and segmentation. The input image, wavelet decomposition, and wavelet reconstruction is as shown in Fig. 2(a), Fig. 2(b) and Fig. 2(c) respectively. 


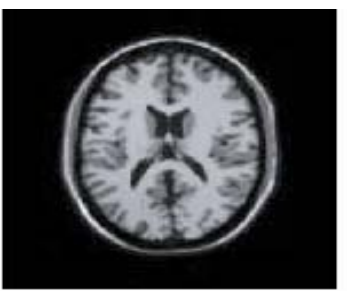

(a)

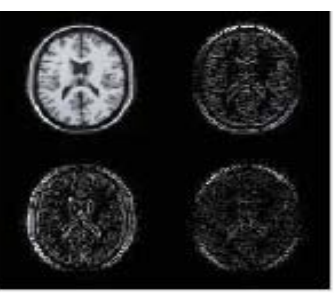

(b)

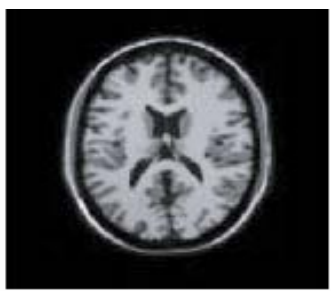

(c)

Fig. 2. a) Input MRI, b) Wavelet decomposition MRI, and c) wavelet reconstructed MRI.

\section{B. Bidimensional Empirical Mode Decomposition(BEMD)}

Empirical mode decomposition (EMD), originally developed by Huang et al. [20], is a data driven signal processing algorithm that has been established to be able to perfectly analyze adaptive, non-linear, and non-stationary data by obtaining local features and timefrequency distribution of the data. The first step of this method is decomposes the data/signal into its characteristic intrinsic mode functions (IMFs), while the second step finds the time frequency distribution of the data from each IMF by utilizing the concepts of Hilbert transform and instantaneous frequency. The complete process is also known as the Hilbert-Huang transform (HHT) [20]. This decomposition technique has also been extended to analyze two-dimensional (2D) data/images, which is known as bidimensional EMD (BEMD).

In this paper, the BEMD has been used to de-noising and decomposition for MR images based on IMFs weighted threshold. After the image decomposed by BEMD method, it was concerned that the images noise mainly distributed in the high and intermediate frequency. These frequency components obtained from the image by applying an algorithm called sifting process.

The sifting procedure decomposes a sampled signal by means of the EMD. The sifting procedure is based on two constraints. Firstly, each IMF has the same number of zero crossings and extrema. Secondly, each IMF is symmetric with respect to the local mean. Furthermore, it assumes that has at least two extrema. Finally, reconstruct the original image to achieve the effect of de-noising. During MR segmentation, it was concerned that the smoothness of the data will influence the segmented quality. The processing example of the BEMD on MRI image is as shown in Fig. 3. In this processing example considered the 48 iteration for IMF function to get the best response.

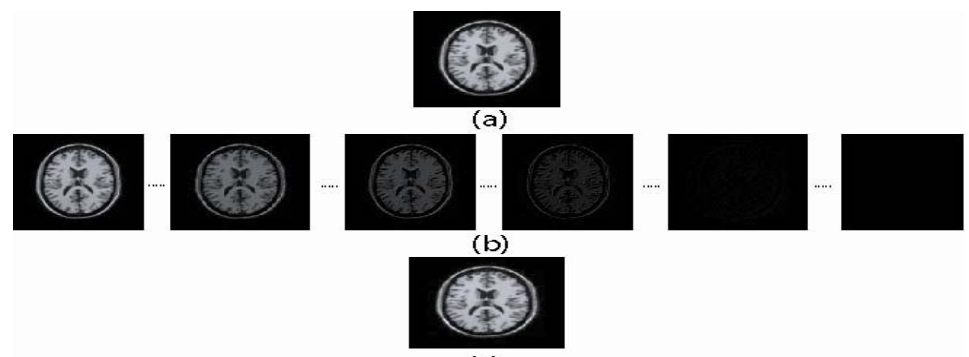

(c)

Fig.3. (a) Input MRI, (b) IMF $(1, \ldots, 6, \ldots, 12, \ldots, 20, \ldots, 48), \ldots$, residue image, and (c) reconstructed Image. 


\section{Fuzzy-C Means}

Fuzzy C-means (FCM) technique is one of the unsupervised clustering techniques used in image segmentation. FCM idea depends on clustering data into two or more classes only by known number of classes. Algorithm is based on minimization of the following objective function:

$$
J\left(U, c_{1}, c_{2}, \ldots, c_{\varepsilon}\right)=\Sigma_{i=1} J_{i}=\sum_{i=1} \Sigma_{j=1} u_{i j} d_{i j}
$$

Here, $u$ is between 0 and $1 ; C_{i}$ is the centroids of cluster $I ; d_{i j}$ is the Euclidean distance between $i^{\text {th }}$ cancroids and $\mathrm{j}^{\text {th }}$ data point; $\mathrm{m} \quad[1, \infty]$ is a weighting function. Fuzzy portioning of known data sample is carried out through an iterative optimization of the objective function:

$$
\begin{aligned}
& u_{i j}=\frac{1}{\sum_{k=1}\left(\frac{d_{i j}}{d_{k j}}\right)^{2 /(m-1)}} \\
& c_{i j}=\frac{\sum_{j=1}^{n} z_{i j}^{m} x_{j}}{\sum_{j=1}^{m} u_{i j}^{m}}
\end{aligned}
$$

This iteration will stop when

$$
\max _{i, j}\left[\left|u_{i j}^{(k+1)}-u_{i j}^{k /}\right|\right]
$$

Where, is a termination criterion between 0 and $1, k$ is the iteration steps. This procedure converges to a local minimum or a saddle point of $J_{m}$.

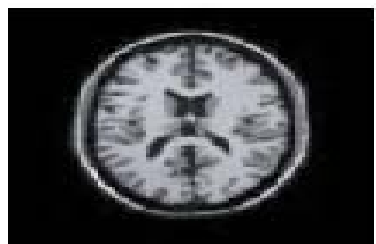

(a)

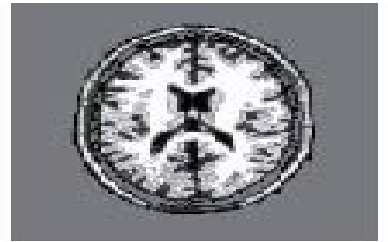

(b)

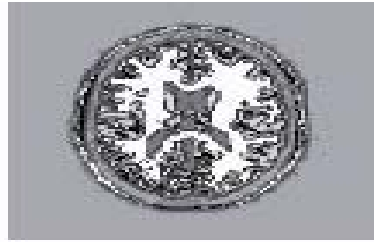

(c)

Fig. 4. a) Input MRI, b) segmented image using Wavelet \& FCM, and c) segmented image using BEMD \& FCM.

\section{EXPERIMENTAL RESULT}

The proposed method is implemented in MATLAB environment and tested on MRI brain web database. The brain web images are simulated MR images generated by the Brain Web simulator with different level of noise $0 \%, 1 \%, 3 \%, 5 \%, 7 \%, 9 \%$ and with different level of INU $0 \%, 20 \%$ and $40 \%$. These images are obtained from Brain Web Database at the McConnell Brain Imaging Centre of the Montreal Neurological Institute, McGill University. An example of images is as shown in Fig. 5(a), 5(b), and 5(c).

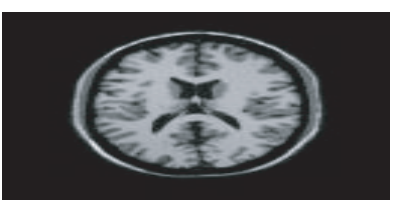

(a)

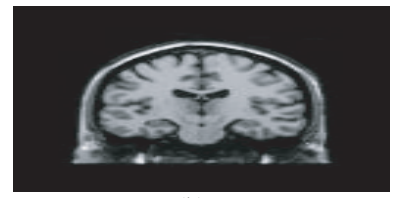

(b)

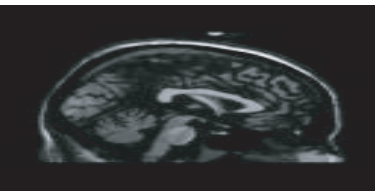

(c)

Fig. 5. (a), (b) and (c) are T1 simulated brain web images. 

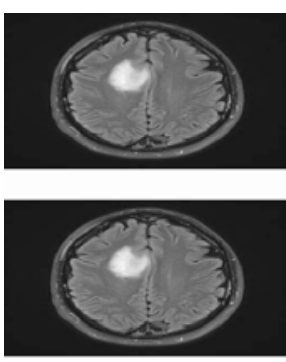

(a)

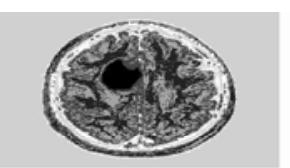

Wavelet \& FCM

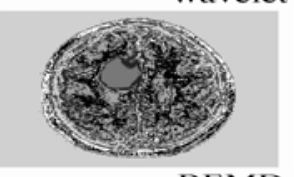

BEMD \& FCM

(b)
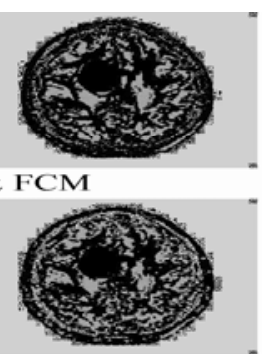

(c)
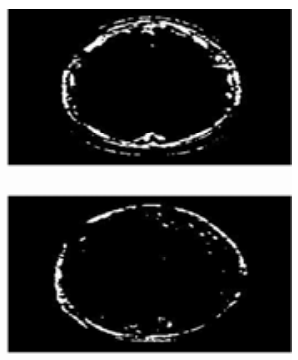

(d)

Fig. 6. Processing example of smooth MRI image for sample 1:(a) original image, (b) segmented image, (c) Gray Matter, and (d) White Matter.
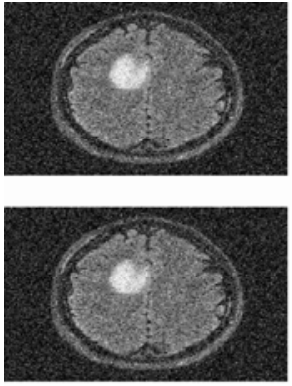

(a)

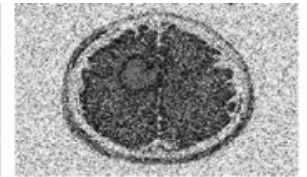

Wavelet \& FCM

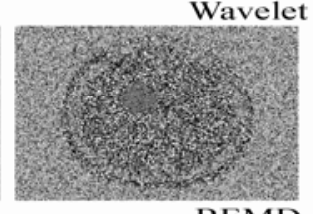

b)
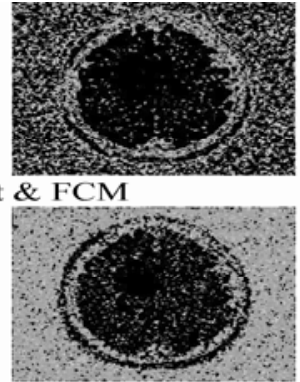

(c)
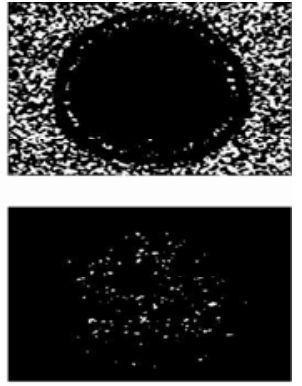

(d)

Fig. 7. Processing example of noisy MRI image for sample 1 (a) original image, (b) segmented image, (c) Gray Matter, and (d) White Matter.
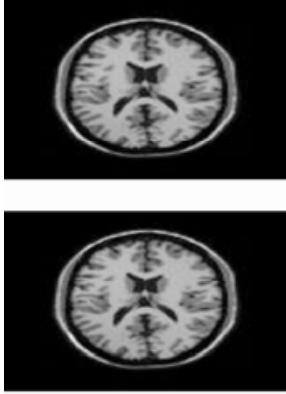

(a)

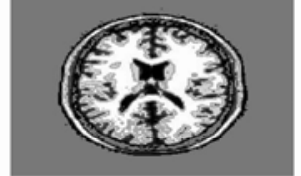

Wavelet \& FCM

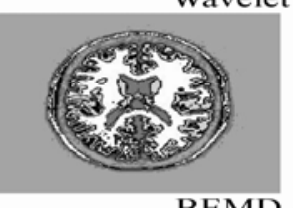

(b)
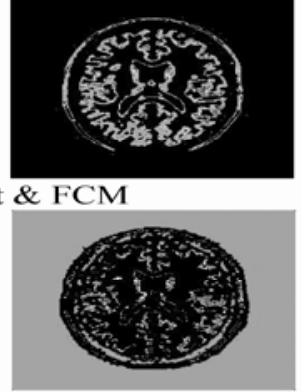

(c)
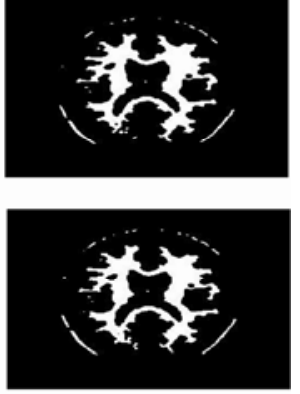

(d)

Fig. 8. Processing example of smooth MRI image for sample 2 (a) original image, (b) segmented image, (c) Gray Matter, and (d) White Matter. 

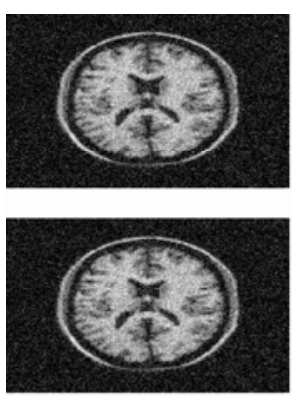

(a)

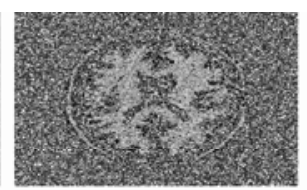

Wavelet

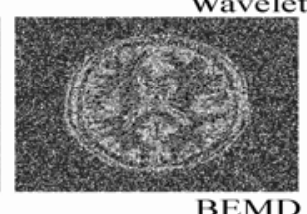

(b)

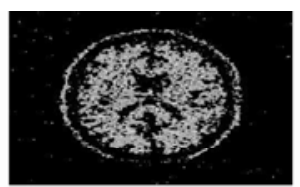

\& FCM

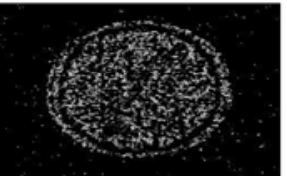

(c)
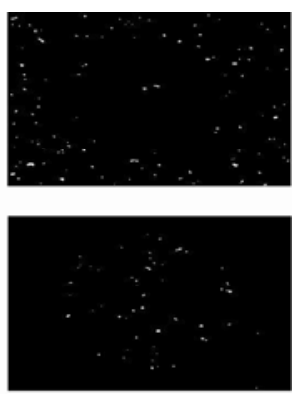

(d)

Fig. 9. Processing example of noisy MRI image for sample 2 (a) original image, (b) segmented image, (c) Gray Matter, and (d) White Matter.
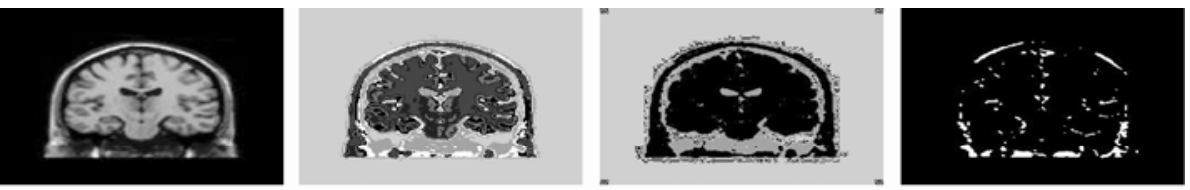

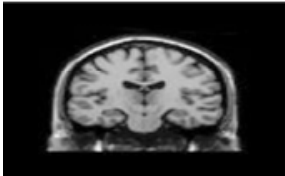

(a)

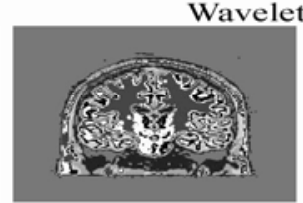

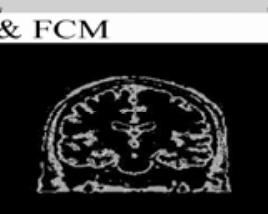

(c)

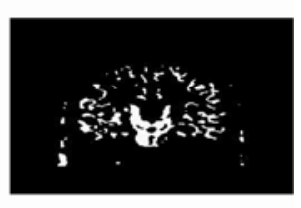

(d)

Fig. 10. Processing example of smooth MRI image for sample 3 (a) original image, (b) segmented image, (c) Gray Matter, and (d) White Matter.
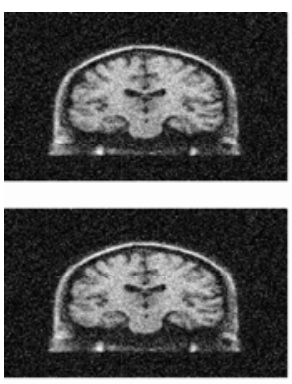

(a)
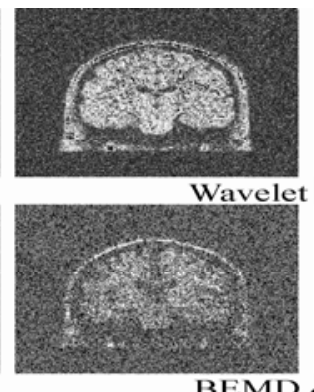

(b)
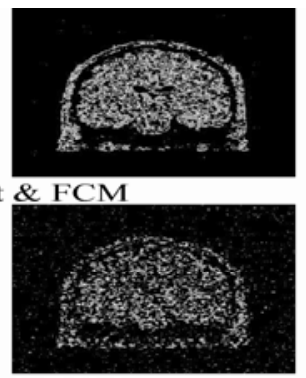

(c)
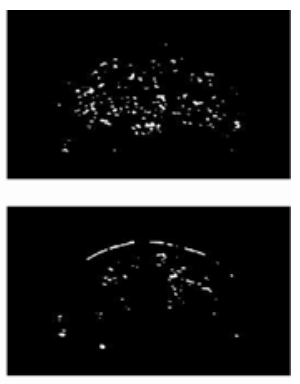

(d)

Fig. 11. Processing example of noisy MRI image for sample 3 (a) original image, (b) segmented image, (c) Gray Matter, and (d) White Matter.

Fig. 6, Fig. 8, and Fig. 10 shows the processing example of sample smooth MRI images applying wavelet and BEMD with FCM clustering. Fig. 7, Fig. 9, and Fig. 11 shows the processing example of sample Gaussian noisy MRI images applying wavelet and BEMD with FCM clustering. 

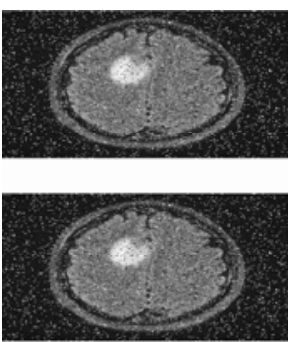

(a)

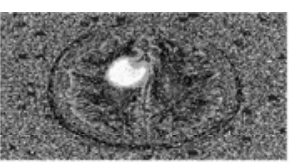

Wavelet

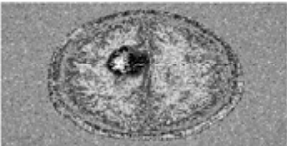

BEMD \& FCM

(b)
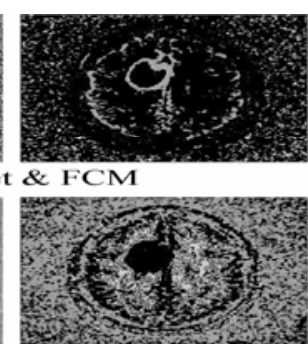

(c)
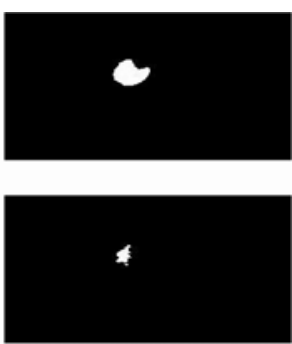

(d)

Fig. 12. Processing example of $10 \%$ Salt \& Pepper noisy MRI image for sample 1 (a) original image, (b) segmented image, (c) Gray Matter, and (d) White Matter.
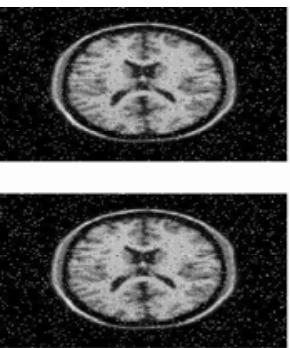

(a)

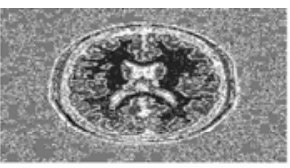

Wavelet

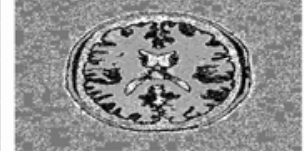

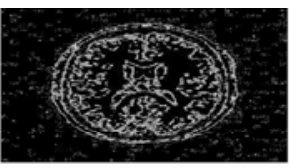

\& FCM

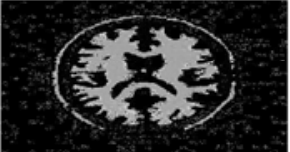

(c)
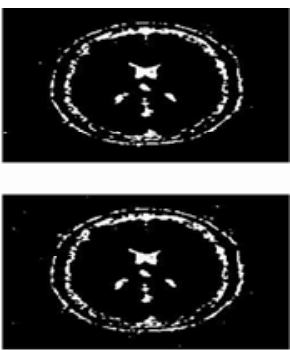

(d)

Fig. 13. Processing example of $10 \%$ Salt \& Pepper noisy MRI image for sample 2 (a) original image, (b) segmented image, (c) Gray Matter, and (d) White Matter.
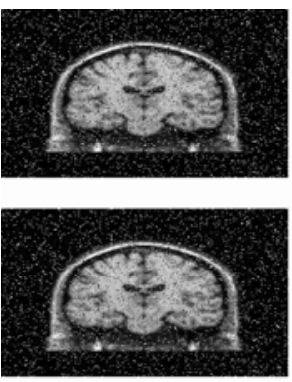

(a)
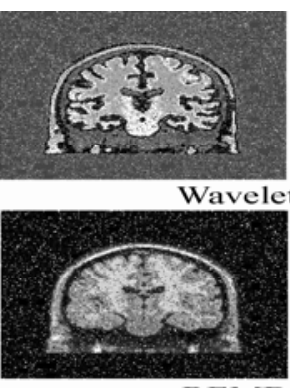
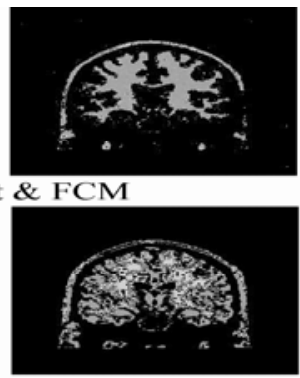

(b)

(c)
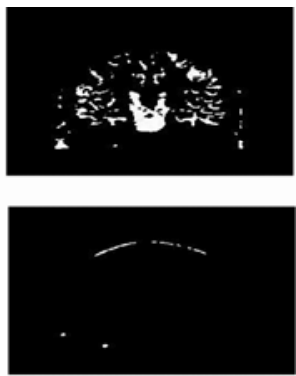

(d)

Fig. 14. Processing example of $10 \%$ Salt $\&$ Pepper noisy MRI image for sample 3 (a) original image, (b) segmented image, (c) Gray Matter, and (d) White Matter.

Fig. 12, Fig. 13, and Fig. 14 shows the processing example of sample Salt \& Pepper noisy MRI images applying wavelet and BEMD with FCM clustering.

The evaluation of the segmentation performance in this paper is measured by the Eq. (5). 
Analyzing Wavelet and Bidimensional Empirical Mode Decomposition of MRI

$$
S N R=\frac{\sum_{y=1}^{N} \sum_{y=a}^{N} f(x, y)^{2}}{\sum_{x=1}^{N} \sum_{y=1}^{N} 5(x y)^{2}}
$$

Where $f(x$, the input is image and $\quad S(x$ is the segmented image.

The SNR value of image sample 1, sample 2, and sample 3 for smooth, adding Gaussian noise and adding $10 \%$ Salt \& Pepper noise is as shown in Table I both for Wavelet and BEMD decomposition method and FCM.

TABLE 1: SNR OF THE INPUT IMAGES

\begin{tabular}{|c|c|c|c|}
\hline \multirow{2}{*}{$\begin{array}{l}\text { Sample } \\
\text { MRI } \\
\text { image }\end{array}$} & \multirow{2}{*}{$\begin{array}{c}\text { Input MRI with } \\
\text { Smooth, Adding } \\
\text { Gaussian and Salt \& } \\
\text { pepper noise }\end{array}$} & \multicolumn{2}{|c|}{ SNR } \\
\hline & & Wavelet \& FCM & BEMD \& FCM \\
\hline \multirow{3}{*}{1} & Smooth & 1.0175 & 1.0977 \\
\hline & $\begin{aligned} \text { mean } & =0 \text { and variance } \\
& =0.025\end{aligned}$ & 0.7749 & 0.7789 \\
\hline & $\begin{array}{c}10 \% \text { Salt \& Pepper } \\
\text { noise }\end{array}$ & 0.8692 & 0.8731 \\
\hline \multirow{3}{*}{2} & Smooth & 0.4249 & 0.4259 \\
\hline & $\begin{aligned} \text { mean } & =0 \text { and variance } \\
& =0.025\end{aligned}$ & 0.6588 & 0.6654 \\
\hline & $\begin{array}{l}10 \% \text { Salt \& Pepper } \\
\text { noise }\end{array}$ & 0.5042 & 0.5241 \\
\hline \multirow{3}{*}{3} & Smooth & 0.3992 & 0.3997 \\
\hline & $\begin{aligned} \text { mean } & =0 \text { and variance } \\
& =0.025\end{aligned}$ & 0.6452 & 0.6472 \\
\hline & $\begin{array}{l}10 \% \text { Salt \& Pepper } \\
\text { noise }\end{array}$ & 0.4955 & 1.0011 \\
\hline
\end{tabular}

The SNR value of Wavelet \& FCM and BEMD \& FCM for sample image 1, sample image 2, and sample image 3 for smooth, adding Gaussian noises and adding $10 \%$ Salt \& Pepper noises are shown in Fig. 15, Fig. 16, and Fig. 17 respectively.

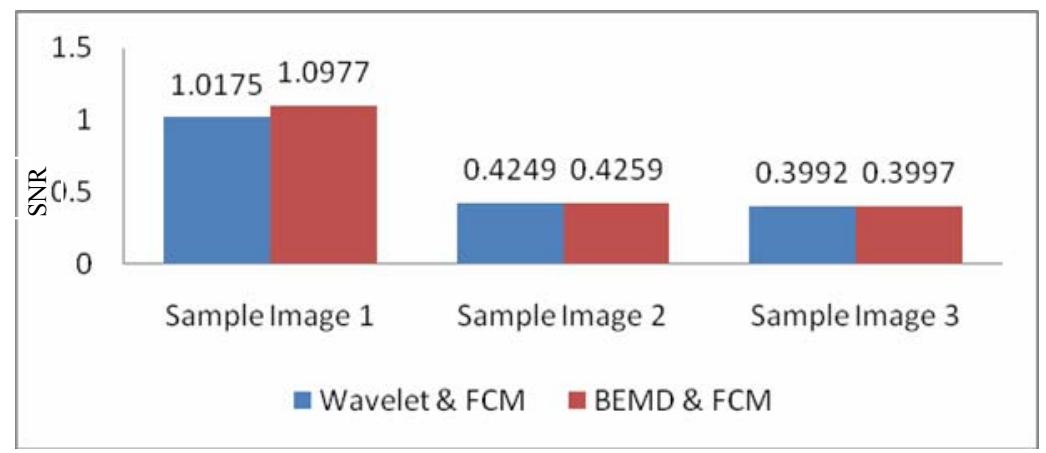

Fig. 15. SNR values of Wavelet \& FCM and BEMD \& FCM for smooth images. 


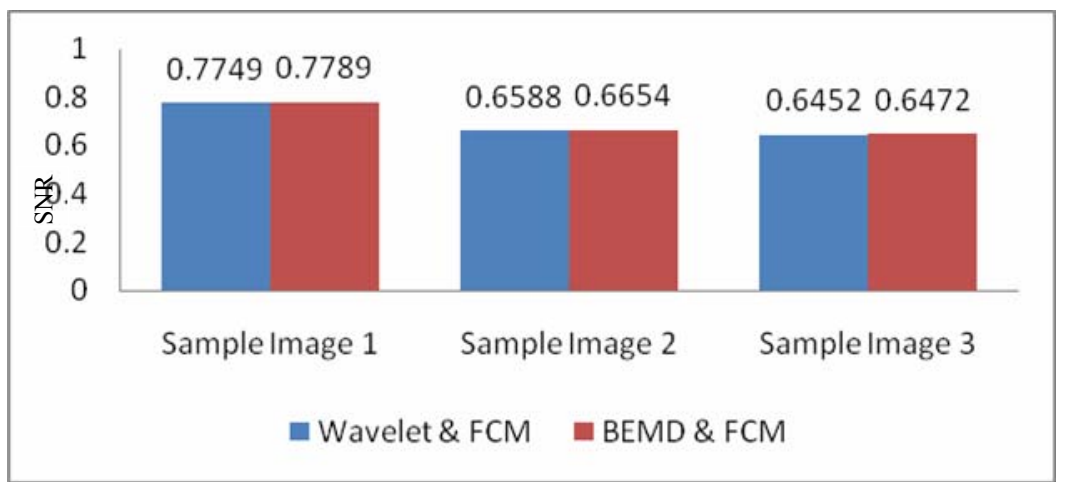

Fig. 16. SNR values of Wavelet \& FCM and BEMD \& FCM for noisy images (Gaussian noise).

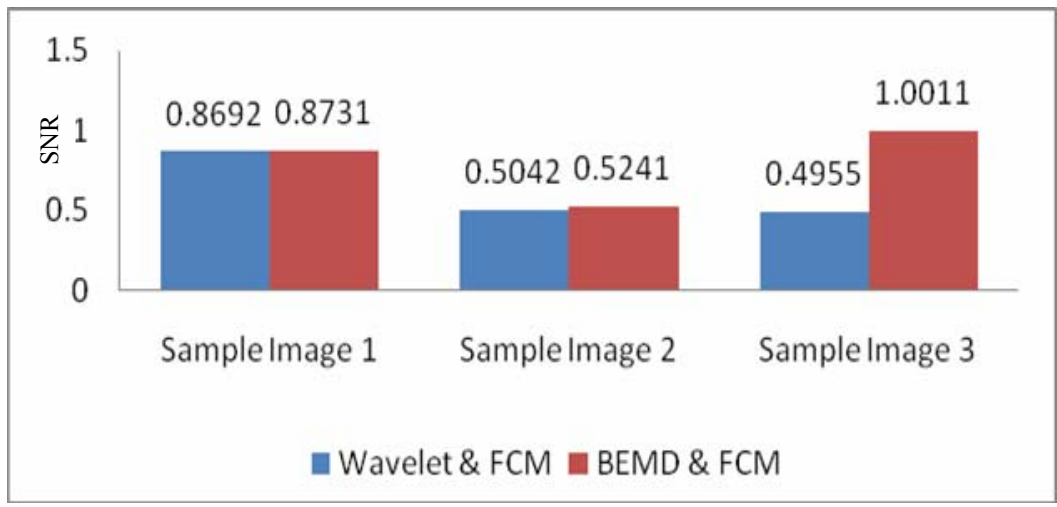

Fig. 17. SNR values of Wavelet \& FCM and BEMD \& FCM for noisy images (10\% Salt \& Pepper noise).

The SNR of the segmented image generated from BEMD and FCM gives the higher value both for smooth and noisy MR Image. So, it should be noted that BEMD decomposition approach is better than the wavelet algorithm for the use of decomposition before applying the segmentation algorithm such as FCM.

\section{CONCLUSIONS}

In this paper presents a robust and efficient approach for the segmentation of medical MR images. For that, wavelet and BEMD decomposition method have been applied in MR images to extract the features. After decompositions, the FCM is use for clustering the image data i.e. generated the segmented image. Then, the best approach is selected by calculating the SNR value. The appropriate approach has been found robust against various medical MR images. The experiments with synthetic Brain Web images have demonstrated the efficiency and robustness of the appropriate approach in segmenting smooth and noisy medical MR images. In future, this works will be improved by increasing overall segmentation performance using another version of FCM for different types of noisy (white noise) MR images. 
Analyzing Wavelet and Bidimensional Empirical Mode Decomposition of MRI

Segmentation using Fuzzy C-Means Clustering

\section{References}

[1] Ouarda, A. S. S. A. S., "MR Brain Real Images Segmentation Based Modalities Fusion and Estimation Et Maximization Approach." International Journal of Advanced Computer Science \& Applications, Vol. 7, No. 1, pp. 267-273, 2016.

[2] Sampath, R. and Saradha, A., "Alzheimer's Disease Image Segmentation with Self-Organizing Map Network," Journal of software, Vol. 10, No. 6, pp. 670-680, 2015.

[3] Sindhuja, A. and Sadasivam, V., "Wavelet based segmentation using optimal statistical features on breast images," ICTACT Journal on Image \& Video Processing, Vol. 4, No. 4, 2014.

[4] Anami, B.S. and Unki, P.H., "A combined fuzzy and level sets based approach for brain MRI image segmentation," Computer Vision, Pattern Recognition, Image Processing and Graphics (NCVPRIPG), 2013 Fourth National Conference on Digital Object Identifier, 10.1109/NCVPRIPG.2013.6776216. pp 1-4, 2013.

[5] Shamsi, H., Seyedarabi, H., and Erfani, S., " MRI image segmentation based on new fuzzy cmeans algorithm," International Journal on Computer Science and Engineering, Vol. 3, No. 8, 2011.

[6]Kouhi, A., Seyedarabi, H., and Aghagolzadeh, A., "A Modified FCM Algorithm for MRI Brain Image Segmentation," In 2011 7th Iranian Conference on Machine Vision and Image Processing, pp. 1-5, IEEE, 2011.

[7] Aboutanos, G. B., Nikanne, J., Watkins, N., and Dawant, B., "Model Creation and Deformation for the Automatic Segmentation of the Brainin MR Images," IEEE Transactions on Biomedical Engineering, Vol. 46, No. 11, 1999.

[8]Karras, D. A., and Mertzios, B.G., "On Edge Detection in Mri Using the Wavelet Transform and Unsupervised Neural Networks", EC-VIP-MC 2003. 4th EURASIP Conference focused on Video I Image Processing and Multimedia Communications, Zagreb, Croatia, 2-5 July 2003.

[9] Gui, L., Lisowski, R., Faundez, T., Huppi, P.S., Lazeyras, F., and Kocher, M., "Automatic Segmentation of Newborn Brain Mri Using Mathematical morphology," In 2011 IEEE International Symposium on Biomedical Imaging: From Nano to Macro, pp. 2026-2030, IEEE, 2011.

[10] Tohka, J. , Dinov, I. D., Shattuck, D.W. , Toga, A.W. ,"Brain MRI tissue classification based on local Markov random fields," Magnetic Resonance Imaging, Volume 28, Issue 4, pp. 557573, May 2010

[11] Birgani, P.M., Ashtiyani, M., and Asadi, S., "MRI Segmentation Using Fuzzy C-means Clustering Algorithm Basis Neural Network," In Proc. ICTTA 2008. 3rd International Conference on Information and Communication Technologies: From Theory to Applications, pp: 1-5, 2008.

[12] Xiao, K., Ho, S. H., and Bargiela, A., "Automatic Brain MRI Segmentation Scheme Based on Feature Weighting Factors Selection on Fuzzy C-means Clustering Algorithms with Gaussian Smoothing," International Journal of Computational Intelligence in Bioinformatics and Systems Biology, Vol. 1, No. 3, pp. 316-331, 2010.

[13] Wang, J., Kong, J., Lu, Y., Qi, M., and Zhang, B., "A Modified FCM algorithm for MRI Brain Image Segmentation Using Both Local and Nonlocal Spatial Constraints," Computerized Medical Imaging and Graphics, Vol. 32, No. 8, pp. 685-698, 2008.

[14] Ardizzone, E., Pirrone, R., and Orazio, O.G., "Fuzzy C-Means Segmentation on Brain MR Slices Corrupted by RF-Inhomogeneity," In Proc. the $7^{\text {th }}$ international workshop on Fuzzy Logic and Applications: Applications of Fuzzy Sets Theory (Berlin, Heidelberg, 2007), WILF '07, SpringerVerlag, pp: 378-384, 2007. 
[15] Mostafa, M.G., Tolba, M.F., Gharib, T.F., and Mageed, M.A., "Medical Image Segmentation Using Wavelet Based Mutiresolution EM Algorithm," In IEEE International Conference of Electronics Technology and Application, IETA, 2001.

[16] Khalifa, I., Youssif, A., and Youssry, H., "MRI brain image segmentation based on wavelet and FCM algorithm," International Journal of Computer Applications, Vol. 47, No. 16, 2012.

[17] Linderhed, A., "Image empirical mode decomposition: A new tool for image processing," Advances in Adaptive Data Analysis, Vol. 1, No. 2, pp. 265-294, 2009.

[18] Nunes, J. C., Bouaoune, Y., Delechelle, E., Niang, O., and Bunel, P., "Image analysis by bidimensional empirical mode decomposition," Image and vision computing, Vol. 21, No.12, pp. 1019-1026, 2003.

[19] Luo, S., "Automated Medical Image Segmentation Using a New Deformable Surface Model," IJCSNS International Journal of Computer Science and Network Security, Vol. 6, No. 5A, pp. 109-115, 2006.

[20] Huang, N. E., Shen, Z., Long, S. R., Wu, M. C., Shih, H. H., Zheng, Q., and Liu, H. H., “ The empirical mode decomposition and the Hilbert spectrum for nonlinear and non-stationary time series analysis," In Proceedings of the Royal Society of London A: Mathematical, Physical and Engineering Sciences, Vol. 454, No. 1971, pp. 903-995,. The Royal Society, 1998. 